PROCEEDINGS OF THE

AMERICAN MATHEMATICAL SOCIETY

Volume 129, Number 1, Pages 1-7

S 0002-9939(00)05528-3

Article electronically published on June 14, 2000

\title{
LICCI IDEALS AND THE NON-ALMOST COMPLETE INTERSECTION LOCUS
}

\author{
MARK R. JOHNSON
}

(Communicated by Wolmer V. Vasconcelos)

\begin{abstract}
We extend results of Huneke and Ulrich on the structure of ideals in the linkage class of a complete intersection, for the class of ideals which are linked to a complete intersection in an even number of steps. In particular for such ideals the non-almost complete intersection locus has codimension at most ten.
\end{abstract}

\section{INTRODUCTION}

Two ideals $I$ and $J$ in a local Gorenstein ring are said to be linked (written $I \sim J)$ if there is a regular sequence $\underline{\alpha}=\alpha_{1}, \ldots, \alpha_{g}$ contained in $I \cap J$ such that $I=(\underline{\alpha}): J$ and $J=(\underline{\alpha}): I$. An ideal $I$ is licci (in the linkage class of a complete intersection) if there is a sequence of links $I \sim I_{1} \sim \cdots \sim I_{n}$, where $I_{n}$ is a complete intersection.

Let $R$ be a regular local ring and let $I$ be a licci $R$-ideal which is not a complete intersection. The work of Huneke and Ulrich 2] completely answers the question of the smoothability of the algebra $R / I$. (An algebra is smoothable in codimension $k$ if it has a deformation satisfying Serre's condition $R_{k}$.) They show that if $R / I$ is not Gorenstein, then $R / I$ is smoothable in codimension 3 , but not in codimension 4 , and if $R / I$ is Gorenstein, then $R / I$ is smoothable in codimension 6 (if the residue field is infinite [3]), but not in codimension 7 . In particular, if $R=k\left[\left[x_{1}, \ldots, x_{k}\right]\right]$ and $R / I$ is a rigid $k$-algebra, then the singular locus of $R / I$ has codimension 4 in the non-Gorenstein case, and codimension 7 in the Gorenstein case. They also show, concerning the non-Gorenstein locus, that if the licci algebra $R / I$ is only Gorenstein in codimension 4, then it must be Gorenstein.

In this paper we prove results concerning the non-almost complete intersection locus. Here we must restrict attention to the evenly licci ideals, those which can be linked to a complete intersection minimally in an even number of steps. This restriction allows us to reduce our problem to the study of the second generic link of a regular ideal. This was the approach of [2], where the singular locus, and well as the non-Gorenstein locus, of this ideal is computed.

Our main contribution is to the computation of the non-almost complete intersection locus of the second generic link of a regular ideal. It turns out that this has two irreducible components, one of codimension 7 (one of the two components of

Received by the editors February 26, 1998 and, in revised form, March 10, 1999.

1991 Mathematics Subject Classification. Primary 13C40, 13H10.

Key words and phrases. Generic link, licci ideal, singular locus.

(C)2000 American Mathematical Society 
the singular locus), and a component of codimension 10. This computation turns out to be much simpler than that for the singular locus. One reason for this is that the new component in codimension 10 is easily seen to be irreducible; the proof of the irreducibility of the components of the singular locus in [2] is much more involved.

In studying evenly licci ideals we must assume that the residue field is infinite; this guarantees that this property is preserved under a faithfully flat morphism ([3]). Under this assumption, we show for an evenly licci ideal (not a complete intersection), the codimension of the non-almost complete intersection locus is at most 10 .

\section{THE SECOND GENERIC LINK OF A REGULAR IDEAL}

In this section we will compute the non-almost complete intersection locus of the second generic link of a regular ideal. We denote by Sing, NCI, NACI, respectively the singular, non-complete intersection, non-Gorenstein, and non-almost complete intersection locus, i.e. the set of primes where the localization is not respectively regular, a complete intersection, Gorenstein, an almost complete intersection. We recall the notation of [2, 3.2] which will be in force throughout this section.

Notation 1.1. Let $R$ be a regular local ring, let $x_{1}, \ldots, x_{g}$ be part of a regular system of parameters of $R(g \geq 3)$, and set $I=\left(x_{1}, . ., x_{g}\right) R$. Let $Y$ be a generic $g \times g$ matrix over $R$, set $T=R[Y]$ and

$$
\left(\begin{array}{c}
\alpha_{1} \\
\vdots \\
\alpha_{g}
\end{array}\right)=Y\left(\begin{array}{c}
x_{1} \\
\vdots \\
x_{g}
\end{array}\right)
$$

We let $K=L_{1}(I)=L_{1}\left(x_{1}, \ldots, x_{g}\right)=\left(\alpha_{1}, \ldots, \alpha_{g}\right) T: I T=\left(\alpha_{1}, \ldots, \alpha_{g}, \Delta\right) T$, where $\Delta$ $=\operatorname{det}(Y)$ (see 1.2), be the first generic link of $I$.

Now let $Z$ be a generic $g \times g+1$ matrix over $T$, set $S=T[Z]=R[Y, Z]$ and

$$
\left(\begin{array}{c}
\beta_{1} \\
\vdots \\
\beta_{g}
\end{array}\right)=Z\left(\begin{array}{c}
\alpha_{1} \\
\vdots \\
\alpha_{g} \\
\Delta
\end{array}\right)
$$

Then $J=L_{2}(I)=\left(\beta_{1}, \ldots, \beta_{g}\right) S: K S$ is the second generic link of $I$.

We will need some further notation. Let $\left(\Delta_{i j}\right)=\operatorname{adj}(Y)$ be the adjoint matrix of $Y$, and set

$$
\begin{gathered}
V=\left(\begin{array}{c}
-x_{1} \\
\operatorname{adj} \mathrm{Y} \\
\vdots \\
-x_{g}
\end{array}\right), \\
U=\left(\begin{array}{c}
Z \\
V
\end{array}\right)
\end{gathered}
$$

let $V_{i}$ be the $i$ th row of $V$, and let

$$
\Delta_{i}=\operatorname{det}\left(\begin{array}{c}
Z \\
V_{i}
\end{array}\right)
$$


Finally, let $W$ be the $g \times g$ submatrix of $Z$ obtained by deleting the last column. We define two $S$-ideals (for $g \geq 3$ )

$$
\begin{gathered}
q_{1}=\left(x_{1}, \ldots, x_{g}, I_{g-1}(Y), I_{g-2}(W Y), I_{g}(W)\right) S, \\
q_{2}=\left(K,\left(I_{g}\left(\begin{array}{c}
Z \\
V_{i}
\end{array}\right) \mid 1 \leq i \leq g\right)\right) S .
\end{gathered}
$$

Proposition 1.2 ([2] 3.1]). (a) $K=\left(\alpha_{1}, \ldots, \alpha_{g}, \Delta\right)$.

(b) $p=\left(I, I_{g-1}(Y)\right) \in \operatorname{Spec}(T), \operatorname{Sing}(T / K)=N C I(K)=V(p)$, and $\operatorname{ecodim}\left((T / K)_{p}\right)=2$.

Proposition 1.3. (a) $J=\left(\beta_{1}, \ldots, \beta_{g}, \Delta_{1}, \ldots, \Delta_{g}\right)=\left(\beta_{1}, \ldots, \beta_{g}, I_{g+1}(U)\right)$.

(b) There is a resolution

$$
0 \longrightarrow S^{g+1} \stackrel{\phi}{\longrightarrow} S^{g(g+3) / 2} \longrightarrow \cdots \longrightarrow S^{2 g} \longrightarrow J \longrightarrow 0,
$$

where

$$
\phi=\left(U^{t} \mid \frac{*}{0}\right),
$$

and the unspecified block denotes a Koszul matrix in $\alpha_{1}, \ldots, \alpha_{g}$.

Proof. Part (a) is shown in [2, 3.3]. To show (b), we compute the resolution of $J$ using the mapping cone construction. It is well-known that there is a resolution

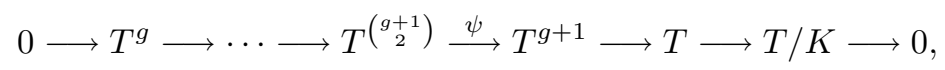

where

$$
\psi=\left(\frac{V}{* \mid 0}\right) .
$$

(The resolution of $K$ may be obtained as well from the Koszul complex on $x_{1}, \ldots, x_{g}$ using the mapping cone construction.) Tensoring this with $S$, we apply the mapping cone construction to the link $K S \sim J$. Since $(\underline{\beta})^{t}=Z(\underline{\alpha}, \Delta)^{t}$, we may choose a lifting $u$ of the inclusion $(\underline{\beta}) S \subset K S$, to be multiplication by $Z$ in degree one. It is then straightforward to check that the last map (after splitting off) is as claimed.

The next theorem of 2] describes the structure of the second generic link of a regular ideal. It is by and large responsible for much of the general structure of licci ideals.

Theorem $1.4([2,3.40])$. (a) $N G(J)=V\left(q_{2}\right)$.

(b) $\operatorname{NCI}(J)=V\left(q_{1}\right) \cup V\left(q_{2}\right)$.

(c) $p_{1}=\sqrt{q_{1}} \in \operatorname{Spec}(S), \operatorname{dim}(S / J)_{p_{1}}=7,(S / J)_{p_{1}}$ is Gorenstein, $d\left((S / J)_{p_{1}}\right)=2$ and $\operatorname{ecodim}\left((S / J)_{p_{1}}\right)=3$.

(d) $p_{2}=\sqrt{q_{2}} \in \operatorname{Spec}(S), \operatorname{dim}(S / J)_{p_{2}}=4, d\left((S / J)_{p_{2}}\right)=1$, and $\operatorname{ecodim}\left((S / J)_{p_{2}}\right)$ $=2$.

Our main result is the following:

Theorem 1.5. Let $p_{3}=\left(x_{1}, \ldots, x_{g}, I_{g-1}(Y), I_{g-1}(Z)\right) S$.

(a) $N A C I(J)=V\left(p_{1}\right) \cup V\left(p_{3}\right)$.

(b) $p_{3} \in \operatorname{Spec}(S), \operatorname{dim}(S / J)_{p_{3}}=10, d\left((S / J)_{p_{3}}\right)=2$, and $\operatorname{ecodim}\left((S / J)_{p_{3}}\right)=2$. 
Proof. We first show (b). It is clear that $p_{3}$ is a prime ideal, and that $h t p_{3}=$ $g+h t I_{g-1}(Y)+h t I_{g-1}(Z)=g+4+6=g+10$. Thus $\operatorname{dim}(S / J)_{p_{3}}=10$. Now consider the resolution of $S / J$ described in Proposition 1.3(b), where $\phi$ denotes the last map. Then the locus $\left\{p \in V(J) \mid r\left((S / J)_{p}\right) \geq r\right\}$, where the type is at least $r$, is given by $V\left(I_{g+2-r}(\phi)\right)$. In particular, $V\left(I_{g-2}(\phi)\right)$ describes the locus where $J$ has type at least 4 . But as $I_{g-2}(Z) \not \subset p_{3}$, it follows that $p_{3} \notin V\left(I_{g-2}(\phi)\right)$, and hence $r\left((S / J)_{p_{3}}\right) \leq 3$. On the other hand, $p_{3}$ contains $I_{g-1}(Y)$; hence $p_{3}$ contains all the entries of the adjoint matrix of $Y$. Thus $p_{3} \in V\left(I_{g-1}(\phi)\right)$, and therefore $r\left((S / J)_{p_{3}}\right) \geq 3$. Hence we have shown that $r\left((S / J)_{p_{3}}\right)=3$.

We now prove that $(S / J)_{p_{3}}$ has embedding codimension 2 . As $(S / J)_{p_{3}}$ is not a complete intersection, we have that $\operatorname{ecodim}\left((S / J)_{p_{3}}\right) \geq 2$. Now choose any sequence of links in $R_{0}\left[x_{1}, x_{2}\right], \tilde{I}_{2} \sim \tilde{I}_{1} \sim \tilde{I}_{0}=\left(x_{1}, x_{2}\right)$, where $R_{0}$ denotes the prime subring of $R$, and $\nu\left(\tilde{I}_{2}\right)=4$, (e.g., one may take $\tilde{I}_{2}=\left(x_{1}, x_{2}\right)^{3}$, and $\left.\tilde{I}_{1}=\left(x_{1}, x_{2}\right)^{2}\right)$ and define $I_{j}=\left(\tilde{I}_{j}, x_{3}, \ldots, x_{g}\right) R$ for $0 \leq j \leq 2$. Then $I_{2} \sim I_{1} \sim I_{0}=I$ is a sequence of links in $R$, and $I_{2}$ is a $(g-2)$-fold hypersurface section. By [2, 2.17], there exists $p \in \operatorname{Spec}(S)$ such that $\left(S_{p}, J_{p}\right)$ is a deformation of $\left(R, I_{2}\right)$. It follows that $\operatorname{ecodim}\left((S / J)_{p}\right) \leq$ $\operatorname{ecodim}\left(R / I_{2}\right)=2$, and that $r\left((S / J)_{p}\right)=r\left(R / I_{2}\right)=d\left(R / I_{2}\right)+1=2+1=3$. It follows that $p \in V\left(I_{g-1}(\phi)\right)$, so in particular $p$ must contain $I_{g-1}(Z)$. On the other hand, $d\left(J_{p}\right)=d\left(\left(I_{2}\right)_{p}\right)=2$, hence $K_{p}$ is not a complete intersection. Thus by Proposition 1.2, $p \in \mathrm{NCI}(K)=V\left(x_{1}, \ldots, x_{g}, I_{g-1}(Y)\right)$, and so $p$ contains both $I$ and $I_{g-1}(Y)$. Hence $p$ contains $p_{3}$, and it follows that $(S / J)_{p_{3}}=\left((S / J)_{p}\right)_{p_{3}}$ has embedding codimension at most 2 . This proves that ecodim $\left((S / J)_{p_{3}}\right)=2$.

As we have seen that $(S / J)_{p_{3}}$ has type 3 and embedding codimension 2 , we may conclude that $d\left((S / J)_{p_{3}}\right)=r\left((S / J)_{p_{3}}\right)-1=3-1=2$. This proves (b).

Now we show (a). To prove one containment, it suffices to check that $p_{1}$ and $p_{3}$ belong to the non-almost complete intersection locus of $J$. This follows by Theorem 1.4(c), and part (b), as $d\left((S / J)_{p_{1}}\right)=2=d\left((S / J)_{p_{3}}\right)$. To prove the other containment, let $p \in \mathrm{NACI}(J)$. We must show that $p$ contains $p_{1}$ or $p_{3}$.

Suppose first that $J_{p}$ is Gorenstein. Then $p \notin \operatorname{NG}(J)=V\left(p_{2}\right)$ and $p \in \operatorname{NCI}(J)=$ $V\left(p_{1}\right) \cup V\left(p_{2}\right)$ by Theorem 1.4. Hence $p \in V\left(p_{1}\right)$ and we are done. Thus we may assume that $r\left((S / J)_{p}\right) \geq 2$. But as we have seen in the proof of (b) above, if $r\left((S / J)_{p}\right) \geq 3$, then $p$ contains $p_{3}$. Thus we may assume that $(S / J)_{p}$ has type exactly 2 .

We first consider the case where $d\left(J_{p}\right) \geq 3$. By appealing again to the resolution of $S / J$ of Proposition 1.3(b), one sees readily from the mapping cone construction that the matrix $Y$ can be taken as a block in a presentation matrix $\psi$ for $J$. Hence $p$ belongs to the locus $\left\{q \in V(J) \mid d\left(J_{q}\right) \geq 3\right\}=V\left(I_{g-2}(\psi)\right)$. Thus $p$ contains in particular the ideal $\left(I, I_{g-2}(Y)\right) S$. But as $p \in \mathrm{NG}(J)=V\left(I_{g}(\phi)\right)$, it follows that $p$ contains $I_{g}(Z)$ as well. Hence $p$ contains $\left(I, I_{g-2}(Y), I_{g}(Z)\right) S \supset$ $\left(I, I_{g-1}(Y), I_{g-2}(W Y), I_{g}(W)\right) S=q_{1}$, as $I_{g-2}(W Y) \subset I_{g-2}(Y)$ ([2, p. 295]). Thus $p \supset \sqrt{q_{1}}=p_{1}$ and we are done in this case.

We have now reduced to the case $r\left((S / J)_{p}\right)=d\left(J_{p}\right)=2$. In this case, we must show that $p$ contains $p_{1}$. We will argue by way of contradiction, assuming now that $p \notin V\left(p_{1}\right)$.

Since $(\underline{\beta})^{t}=Z(\underline{\alpha}, \Delta)^{t}$, modulo $\Delta=\operatorname{det}(Y)$, we have that $(\underline{\beta})^{t}=W(\underline{\alpha})^{t}=$ $W Y(\underline{x})^{t}$. But since $p \not \supset p_{1}$, it follows that $I_{g-2}(W Y) \not \subset p$. Hence, after changing generators, modulo $\Delta, g-2$ elements of $\beta_{1}, \ldots, \beta_{g}$ generate a regular ideal, say $x_{1}, \ldots, x_{g-2}$, locally at $p$. In particular, $(J, \Delta)_{p}$ is a $(g-2)$-fold hypersurface section. 
Thus if we set $T=S_{p}$, we have that $(J, \Delta) T=\left(x_{1}, \ldots, x_{g-2}, L\right)$, where $L$ is a perfect $T$-ideal of grade 3 , necessarily with $d(L)=r(T / L)=2$, and $x_{1}, \ldots, x_{g-2}$ is regular on $T / L$. Note that $\Delta$ is a regular parameter for $T$. Indeed, otherwise $J$ would have to contain $g-2$ elements which are part of a regular system of parameters of $T$, and in particular would be a $(g-2)$-fold hypersurface section, which is not the case.

Now as $J \sim K$, the universal link 2] $J T(X) \sim L^{1}(J T)$ implies that $L^{1}(J T)$ is an almost complete intersection. But on the other hand, $\left(L^{1}(J T), \Delta\right)=L^{1}((J, \Delta) T)=$ $L^{1}\left(x_{1}, \ldots, x_{g-2}, L\right)=\left(L^{1}(L), x_{1}, . ., x_{g-2}\right)$, so $L^{1}(L)$ is an almost complete intersection as well. It follows that $L$ (or at least $L T(X)$ ) belongs to a class of grade 3 , type 2 ideals studied by A. Brown [1, 2.5]. To complete the proof, note that by [4, 2.6 or 3.2 ], it follows that $J T$, and hence $L$, is an almost complete intersection in codimension 7 . We will obtain the required contradiction by showing directly that $L$ is not an almost complete intersection is codimension 7 .

By the structure theorem [1, 4.4], there is a $5 \times 5$ alternating matrix $T=\left(t_{i j}\right)$, with $t_{12}=0$, and nonunits $z_{1}$ and $z_{2}$ such that $L=\left(T_{1}, T_{2}, z_{1} T_{123}+z_{2} T_{3}, z_{1} T_{124}+\right.$ $z_{2} T_{4}, z_{1} T_{125}+z_{2} T_{5}$ ). Here $T_{i_{1} \cdots i_{k}}$ denotes the (signed) pfaffian of the alternating submatrix of $T$ obtained by deleting rows and columns $i_{1}, \ldots, i_{k}$. The resolution of this ideal is computed in [1, 3.6]; one sees immediately that the entries of the presentation matrix generate the ideal $\left(I_{1}(T), z_{1}\right)$.

Since $L$ has deviation two, the non-almost complete intersection locus of $L$ is defined by the entries of the presentation matrix; hence $\operatorname{NACI}(L)=V\left(I_{1}(T), z_{1}\right)$. Thus codim $(\operatorname{NACI}(L))=h t\left(I_{1}(T), z_{1}\right)-h t L \leq 9+1-3=7$. This shows that $L$ is not an almost complete intersection in codimension 7 , and completes the proof of the theorem.

\section{EVENLY LICCI IDEALS}

In this section we apply the results on the second generic link of a regular ideal of the previous section, to establish a kind of structure theorem. We first need to refine the notion of a licci ideal.

Definition 2.1. Let $R$ be a local Gorenstein ring and let $I$ be a licci $R$-ideal. We say that $I$ is evenly licci (respectively, oddly licci) if the smallest number of steps that $I$ can be linked to a complete intersection is even (respectively, odd).

We will see that the methods of [2] can still be employed to prove some further results for the class of evenly licci ideals.

The next observation follows immediately using [3, 2.4] (cf. [2, 2.23]).

Remark 2.2. Let $R$ be a regular local ring with infinite residue field and let $I$ be a licci $R$-ideal.

(a) If $I$ is Gorenstein, then $I$ is evenly licci.

(b) If $I$ is an almost complete intersection (and not a complete intersection), then $I$ is oddly licci.

We recall the following theorem, which is one of the main results of [2] on the structure of licci ideals. One says that $(S, J)$ is essentially a deformation of $(R, I)$ if $(S, J)$ may be obtained from $(R, I)$ by deformation, localization, or residue field extension (or descent).

Theorem 2.3 ([2, 4.3]). Let $R$ be a regular local ring and let $I$ be a licci $R$-ideal which is not a complete intersection. Then $(R, I)$ has essentially a deformation 
$(S, J)$ such that either

(a) $S / J$ is Gorenstein, $d(S / J)=2, \operatorname{ecodim}(S / J)=3, \operatorname{codim}(N C I(S / J))=7$, or

(b) $d(S / J)=1, \operatorname{ecodim}(S / J)=2, \operatorname{codim}(N C I(S / J))=4$.

The significance of this theorem lies in the fact that it reduces certain questions about licci ideals to the simplest nontrivial examples, namely the $2 \times 2$ minors of a generic $2 \times 3$ matrix, and the $4 \times 4$ pfaffians of a generic $5 \times 5$ alternating matrix. We now obtain a stronger theorem for evenly licci ideals, showing that we may essentially deform to the simplest evenly licci algebras:

Theorem 2.4. Let $R$ be a regular local ring with infinite residue field and let $I$ be an evenly licci $R$-ideal which is not a complete intersection. Then $(R, I)$ has essentially a deformation $(S, J)$ such that either

(a) $S / J$ is Gorenstein, $d(S / J)=2, \operatorname{ecodim}(S / J)=3, \operatorname{codim}(N C I(S / J))=7$, or

(b) $d(S / J)=2, \operatorname{ecodim}(S / J)=2, \operatorname{codim}(N C I(S / J))=4$.

Proof. This follows by the proof of Theorem 4.3 in [2], by now replacing Theorem 1.4 with Theorem 1.5 and using Remark 2.2.

Corollary 2.5. Let $R$ be a regular local ring with infinite residue field and let $I$ be an evenly licci $R$-ideal which is not a complete intersection. Then $R / I$ is not an almost complete intersection is codimension 10.

Proof. By Theorem 2.4, it is enough to show that the rings described in parts (a) and (b) are not almost complete intersections in codimension 10, which is wellknown.

We next give a somewhat stronger version of Theorem 2.4. Note that in case $I$ is Gorenstein, then it is necessarily the case in Theorem 2.4 (and Theorem 2.3) that $(R, I)$ has essentially a deformation to a pair $(S, J)$ described in part (a). In 5 . 2.6], it is shown that conversely if $I$ is not Gorenstein, then $(R, I)$ has essentially a deformation $(S, J)$ as described in part (b) of Theorem 2.3. We can show a similar improvement of Theorem 2.4, depending on the type of a certain universal link $L^{i}(I)$ of $I([2])$.

Theorem 2.6. Let $R$ be a regular local ring with infinite residue field, let $I$ be an evenly licci $R$-ideal which is not a complete intersection, and let $n$ be the smallest (even) positive integer such that $I$ is linked to a complete intersection in $n$ steps. Set $r=r\left(R(X) / L^{n-2}(I)\right)$.

(a) If $r \neq 3$, then $(R, I)$ has essentially a deformation $\left(S_{1}, J_{1}\right)$ with $S_{1} / J_{1} \cong$ $\left(P[X] / P f_{4}(X)\right)_{\left(m_{P}, X\right)}$, where $P$ is a regular local ring and $X$ is a generic alternating $5 \times 5$ matrix.

(b) If $r=3$, then $(R, I)$ has essentially a deformation $\left(S_{2}, J_{2}\right)$ with $S_{2} / J_{2} \cong$ $\left(P[X] / I_{3}(X)\right)_{\left(m_{P}, X\right)}$, where $P$ is a regular local ring and $X$ is a generic $3 \times 4$ matrix.

Proof. In the notation of the proof of Theorem 2.3 ([2, Proof of 4.3]), it will be sufficient to show that $q \supset p_{1}$ in case (a), and that $q \supset p_{3}$ in case (b), where $\operatorname{NACI}\left(L_{2}\left(I_{2}\right)\right)=V\left(p_{1}\right) \cup V\left(p_{3}\right)$.

If $r=3$, then the proof of Theorem 1.5 shows that $q$ contains $p_{3}$, which proves (b). On the other hand, if $r \neq 3$, then the proof of Theorem 1.5 also shows that $q$ contains $p_{1}$. This is shown explicitly when $r \leq 2$; in case $r \geq 4$ we have that $q \in V\left(I_{g-2}(\phi)\right) \subset V\left(I_{g-2}(Z)\right)$, hence $q \supset\left(I, I_{g-1}(Y), I_{g-2}(Z)\right) \supset$ $\left(I, I_{g-1}(Y), I_{g-2}(W)\right) \supset\left(I, I_{g-1}(Y), I_{g-2}(W Y), I_{g}(W)\right)=q_{1}$. 
As an application, we can now show that "most" evenly licci algebras have a codimension seven non-almost complete intersection locus.

Corollary 2.7. Let $k$ be an infinite field, let $R=k\left[\left[x_{1}, . ., x_{n}\right]\right]$, let $I$ be an evenly licci $R$-ideal satisfying part (a) of Theorem 2.6, and assume that $R / I$ is rigid over $k$, but not regular. Then codim $(N A C I(R / I))=7$.

Proof. The lower bound follows from [4,3.2], while the upper bound is given by Theorem 2.6.

\section{REFERENCES}

[1] A. Brown, A structure theorem for a class of grade three perfect ideals, J. Algebra 105 (1987), 308-327. MR 88a:13019

[2] C. Huneke and B. Ulrich, The structure of linkage, Annals Math. 126 (1987), 277-334. MR 88k:13020

[3] C. Huneke and B. Ulrich, Algebraic linkage, Duke Math. J. 56 (1988), 415-429. MR 89e:13023

[4] C. Huneke and B. Ulrich, Local properties of licci ideals, Math. Z. 211 (1992), 129-154. MR 93j:13018

[5] B. Ulrich, "Theory and applications of universal linkage", in Commutative Algebra and Combinatorics, Advanced Studies in Pure Mathematics 11, North Holland, Amsterdam, 1987, p. 285-301. MR 89k:13022

Department of Mathematical Sciences, University of Arkansas, Fayetteville, ARKANSAS 72701

E-mail address: mark@math.uark.edu 